\title{
Group actions on Jacobian varieties
}

Anita M. Rojas

\begin{abstract}
Consider a finite group $G$ acting on a Riemann surface $S$, and the associated branched Galois cover $\pi_{G}: S \rightarrow Y=S / G$. We introduce the concept of geometric signature for the action of $G$, and we show that it captures much information: the geometric structure of the lattice of intermediate covers, the isotypical decomposition of the rational representation of the group $G$ acting on the Jacobian variety $J S$ of $S$, and the dimension of the subvarieties of the isogeny decomposition of $J S$. We also give a version of Riemann's existence theorem, adjusted to the present setting.
\end{abstract}

\section{Introduction}

We study the decomposition of Jacobians endowed with a group action, the objective being to find equivariant decompositions of Jacobians into factors of geometric significance [3]. Part of our study follows the line presented in [8], where Ksir finds, in the case of a finite group $G$ acting on a Riemann surface $S$, the isotypical decomposition of the analytical representation for the action of $G$ on the corresponding Jacobian variety. This is accomplished in [8] for groups whose rational irreducible representations are absolutely irreducible.

We consider any finite group $G$, and use both representation theory and group actions to find the isotypical decomposition of the rational representation of the action of $G$ on the Jacobian variety. We compute the dimension of the subvarieties in the $G$-equivariant decomposition of the Jacobian variety, in the sense of [3], Theorem 2.1. These results are obtained in terms of the geometric signature for the action of $G$ on $S$, which generalizes the usual signature [2]. We give a definition in Section 2.

2000 Mathematics Subject Classification: 14H40, 14L30.

Keywords: Jacobian varieties, Riemann surfaces, group actions, Riemann's existence theorem, geometric signature. 
We consider $S$ a closed Riemann surface with an action of a finite group $G$. This induces an action of $G$ on the Jacobian variety of $S$, denoted $J S$, whose rational representation $\rho_{\mathbb{Q}}$ is given by the action of $G$ on the rationalization $H_{1}(S, \mathbb{Z}) \otimes \mathbb{Q}$ of the first homology group of $S$. In order to obtain the isotypical decomposition of $\rho_{\mathbb{Q}}$, we study the action of $G$ on $J S$ through the action on the corresponding Riemann surface $S$.

First we show that the geometric signature reflects the complete geometric structure of the lattice of intermediate covers. In this regard, we prove in Section 3

Theorem 3.7 Let $S$ be a Riemann surface with $G$-action. Then there is a bijective correspondence between geometric structures for the lattice of the subgroups of $G$ and geometric signatures for the action of $G$.

The fact that the geometric signature captures the information about the intermediate covers allows us to implement an algorithm, supported by G.A.P [6], which gives for any finite group $G$ and any subgroup $H$ of $G$, the signature for the action of $H$ on $S$; the genus of $S / H$ and the branch points of $\pi_{H}: S \rightarrow S / H$ (associating them with the branch points of $\pi_{G}: S \rightarrow S / G$ ). And the cycle structure of the other covering, which is not necessarily Galois, $\pi^{H}: S / H \rightarrow S / G$ over each branch point of $\pi_{G}$.

We also study, in Section 4, the existence of a Riemann surface with an action of a group $G$ with a given geometric signature. That is, we adapt Riemann's existence theorem to the setting of geometric signature (see Theorem 4.1). We remark that in [18], H. Völklein proved similar results, but restricted to the case in which the quotient by the $G$-action is the Riemann sphere. We allow any genus for the quotient. Finally, we use the geometric signature to find an equivariant decomposition for the rational representation of the group $G$ acting on $J S$ (Theorem 5.10), and to find the dimension of each subvariety in this isogeny decomposition of $J S$ (Theorem 5.12). As a corollary, we obtain that all of these dimensions are positive if the genus of the orbit surface $S / G$ is greater or equal than 2 , which was obtained by different methods in [9], and conditions characterizing the situation when the orbit surface has genus 1 . We also present several examples of the geometric signature and contrast it with the usual signature.

Acknowledgments. This article is part of my PhD. thesis written under Professor Rubí Rodríguez at the Pontificia Universidad Católica de Chile. I am very grateful to Professor Rodríguez for sharing, patiently and kindly, her time, knowledge and experience. I would also like to thank: Fundación Andes for generous financial support during my PhD. studies, Professors A. Carocca for helpful advice, and E. Friedman, who helped me to present this work. I also express my acknowledgments to the ICTP, where the final 
version of the paper was written. Finally, I have special words of gratitude to Professor Sevín Recillas-Pishmish for sharing his knowledge and, most of all, for his constant concern to encourage and support young mathematicians.

\section{Preliminaries}

In this section we set up the notation and definitions used throughout this work, as well as recall some known results. In this paper, a Riemann surface is a connected, compact, one-dimensional complex manifold without boundary, i.e. all surfaces here are closed.

The number of elements of a (finite) group $G$ is denoted by $|G|$, and the index of a subgroup $H$, by $[G: H]$. An action of a group $G$ on a Riemann surface $S$ is given by a monomorphism of the group $G$ onto a subgroup of the analytical automorphism group $\operatorname{Aut}(S)$ of $S$. We will refer to the "automorphism" $g$ in $G$. The branched cover associated to the $G$-action will be denoted by $\pi_{G}: S \rightarrow S / G$. The orbit of a point $p \in S$ by the subgroup $H \leq G$ is denoted by $\mathbb{O}_{p}^{H}=\{y \in S: y=g(p)$ for some $g \in H\}$, and the stabilizer in $H$ of $p \in S$ is the cyclic $([1, \S 3.10$, p. 17]) subgroup of $H$ denoted by $H_{p}=\{g \in H: g(p)=p\}$.

We introduce the concept of geometric signature for the action of $G$ on $S$ as a natural fusion between the known definitions of signature or branching data [2] and of type of a branch value [13], which we now recall.

A branched covering $f: X \rightarrow Y$, between Riemann surfaces $X$ and $Y$, is by definition a surjective holomorphic map (in particular, non-constant). A point in $X$ is a branch point for $f$ if $f$ fails to be locally one-to-one in there. The image of a branch point is a branch value of $f$. Let $B$ be the set of branch values of $f$. For $q \in B$ consider its fiber $f^{-1}(q)=\left\{p_{1}, \ldots, p_{s}\right\} \subset X$. Then the cycle structure of $f$ at $q$ is the $s$-tuple $\left(n_{1}, \ldots, n_{s}\right)$ where $n_{j}$ is the ramification index of $f$ at $p_{j}$. That is, $f$ is $n_{j}$-to- 1 at $p_{j}, n_{j}>1$.

Now let $S$ denote a closed Riemann surface with $G$ a group of conformal automorphisms of $G$. Let $\left\{p_{1}, \ldots, p_{t}\right\} \subset S$ be a maximal collection of nonequivalent branch points with respect to $G$ (i.e. the $p_{j}$ are in different $G$-orbits). For each $j=1, \ldots, t$, consider the stabilizer $G_{j}$ of $p_{j}$. The signature or branching data [2] of $G$ on $S$ for the cover $\pi_{G}: S \rightarrow S / G$ is the tuple $\left(\gamma ; m_{1}, \ldots, m_{t}\right)$, where $\gamma$ is the genus of $S / G$ and $m_{j}=\left|G_{j}\right|$ for each $j$. Note that $0 \leq t \leq 2 \gamma+2$, and that there are restrictions on $|G|$ and on the branching data that can occur given by Riemann-Hurwitz formula

$$
g=|G|(\gamma-1)+1+\frac{|G|}{2} \sum_{j=1}^{t}\left(1-\frac{1}{m_{j}}\right),
$$

where $g$ denotes the genus of $S$, and $\gamma$ that of $S / G$. We say that the point $\pi_{G}\left(p_{j}\right) \in S / G$ is marked with the number $m_{j}$. 
Consider now $G_{j}$ a (non-trivial) cyclic subgroup of $G$. According to [13] a branch value $q \in S / G$ is called of type $G_{j}$, if $G_{j}$ is the stabilizer of at least one point in the fiber of $q$. It is not difficult to show that if there is a point $p \in S$ with non-trivial stabilizer $G_{p}$, then the points in its orbit have stabilizers running through the complete conjugacy class of $G_{p}$. Hence we will call $q \in S / G$ of type $C_{j}$ if the stabilizer of the points in its fiber are the elements of the (complete) conjugacy class $C_{j}$ of $G_{j}$. For the computations developed in the following sections, it is not critical to know all the conjugacy classes of cyclic subgroups of $G$. The type of the branch values can be given by a cyclic subgroup $G_{j}$ instead of a conjugacy class.

As above, let $S$ be a closed Riemann surface and $G$ a group of conformal automorphisms of $S$. Let $\left\{q_{1}, \ldots, q_{t}\right\} \subset S / G$ be a maximal collection of branch values for the covering $\pi_{G}: S \rightarrow S / G$. We define the geometric signature of $G$ on $S$ as the tuple $\left(\gamma ;\left[m_{1}, C_{1}\right], \ldots,\left[m_{t}, C_{t}\right]\right)$, where $\gamma$ is the genus of $S / G, C_{j}$ is the type of the branch value $q_{j}$ and $m_{j}$ is the order of any subgroup in $C_{j}$. It is clear that the $m_{j}$ are unnecessary, given the $C_{j}$, but we keep them in order to show that this concept is a generalization of the usual signature.

Consider now a lattice of subgroups of $G$. By the geometric structure of the lattice we mean the complete information about all the intermediate quotients by each subgroup of the lattice; that is, the signature of the covering $\pi_{H}: S \rightarrow S / H$, and the cycle structure for the covering $\pi^{H}: S / H \rightarrow S / G$ for each $H$ in the lattice.

We need some known results of representation theory and group actions on abelian varieties, that we include next. We refer to [4], where the following definitions and results may be found. We denote $\operatorname{Irr}_{F}(G)$ the set of irreducible representations of $G$ up to isomorphism, over the field $F$. If $\rho \in \operatorname{Irr}_{\mathbb{C}}(G)$, then $\rho: G \rightarrow G L(U)$, where $U$ is a finite-dimensional complex vector space. For all subgroups $H$ of $G, \operatorname{Fix}_{H}(U)$ denotes the set of fixed points of $U$ under the action of $H$; and for all subspaces $V$ of $U, \operatorname{dim}(V)$ is the complex dimension of $V$. We let $L_{U}$ denote the field of definition of $U$ and let $K_{U}=\mathbb{Q}\left(\chi_{U}(g): g \in G\right)$ denote the field obtained by adjoining to the rational numbers $\mathbb{Q}$ the values of the character $\chi_{U}$. Then $K_{U} \subseteq L_{U}$ and $\ell_{U}=\ell_{\mathbb{Q}}(U)=\left[L_{U}: K_{U}\right]$ is the Schur index of $U$. As usual, we abuse notation by identifying the representation with the underlying vector space.

The next result states the relation between $\mathbb{Q}$-representations and the $G$-equivariant isogeny decomposition of an abelian variety with $G$ action, in terms of the (abstract) group $G$ involved.

Theorem 2.1. ([9], [3]) Let $G$ be a finite group acting on an abelian variety $A$. Let $\mathcal{W}_{1}, \ldots, \mathcal{W}_{r}$ denote the irreducible $\mathbb{Q}$-representations of $G$ up to isomorphism, and $s_{j}=\operatorname{dim}\left(U_{j}\right) / \ell_{j}$, where $U_{j}$ is a complex irreducible 
representation associated to $\mathcal{W}_{j}$ and $\ell_{j}$ is the Schur index of $U_{j}$.

Then there are abelian subvarieties $B_{1}, \ldots, B_{r}$, such that each $B_{j}^{s_{j}}$ is $G$-stable and associated to the representation $\mathcal{W}_{j}$, and there is a $G$-equivariant isogeny $A \sim B_{1}^{s_{1}} \times \cdots \times B_{r}^{s_{r}}$.

It is important to remark that some of the varieties $B_{j}$ 's appearing in the Theorem may be of dimension zero for some particular $G$-actions. For instance, in the case of $A$ being the Jacobian of a Riemann surface $S$ with $G$ action, the variety $B$ corresponding to the trivial representation may be taken as the Jacobian of the Riemann surface $S / G$, whose genus may be equal to zero.

\section{Intermediate covers}

In this section we describe the geometric structure of the lattice of intermediate quotients (see Section 2) in terms of the geometric signature.

Let $S$ be a surface with $G$-action, consider $p$ a point in $S$ and $\mathbb{O}_{p}^{G}$ its orbit. It is clear that the orbit $\mathbb{O}_{p}^{G}$ has cardinality $\sharp \mathbb{O}_{p}^{G}=\left[G: G_{p}\right]$, where $G_{p}$ is the stabilizer in $G$ of $p$. Moreover, $q \in \mathbb{O}_{p}^{G}$ if and only if there exists $h \in G$ such that $q=h(p)$. Then $G_{p}^{h^{-1}}:=\left\{h g h^{-1}: g \in G_{p}\right\}=G_{q}$. By definition, to make packages in $\mathbb{O}_{p}^{G}$ (or to pack $\mathbb{O}_{p}^{G}$ ) is to group the points in $\mathbb{O}_{p}^{G}$ into disjoint subsets, such that each subset consists of all the points sharing the same subgroup of $G$ as stabilizer. Each one of these subsets is called a package, and the stabilizer associated to a package is the stabilizer of the points in it. Hence on any orbit, each package is formed by points with the same stabilizer; two different packages have different, albeit conjugate, stabilizers.

Before stating our next result, let us fix some notation: $N_{G}(H)$ denotes the normalizer in $G$ of the subgroup $H$, a left transversal of a subgroup $H$ is a set of elements of $G$ which are representatives of the left cosets of $H$ in $G$, and $H^{l^{-1}}:=\left\{l g l^{-1}: g \in H\right\}$. The proof of the following Lemma is straightforward.

Lemma 3.1. Given $S$ a Riemann surface with $G$-action, let $p$ be a branch point in $S$. Then

1. In $\mathbb{O}_{p}^{G}$ there are $\left[G: N_{G}\left(G_{p}\right)\right]$ packages. The stabilizer of any point in a package in $\mathbb{O}_{p}^{G}$ is conjugate to $G_{p}$ by an element of a left transversal of its normalizer $N_{G}\left(G_{p}\right)$. Conversely, for any element $l$ of a left transversal of $N_{G}\left(G_{p}\right)$ there is a package in $\mathbb{O}_{p}^{G}$ whose points are stabilized by $G_{p}^{l^{-1}}$.

2. Every package has $\left[N_{G}\left(G_{p}\right): G_{p}\right]$ points. 


\subsection{Signature for the covering $\pi_{H}: S \rightarrow S / H$}

Let $S$ be a Riemann surface with $G$-action and let $H$ be any subgroup of $G$. As the covering $\pi_{H}$ is Galois, its structure is described by giving the signature for the action of $H$. For this we need to compute the genus of $S / H$ and the branch values of $\pi_{H}$ in terms of the geometric signature for the action of $G$. We first describe the genus of $S / H$ as follows.

Proposition 3.2. Let $S$ be a Riemann Surface with $G$-action of geometric signature $\left(\gamma ;\left[m_{1}, C_{1}\right], \ldots,\left[m_{t}, C_{t}\right]\right)$. Then for each subgroup $H \leq G$ the genus of $S / H$ is given by

$$
g_{S / H}=[G: H](\gamma-1)+1+\frac{1}{2} \sum_{j=1}^{t} \sum_{l \in \Omega_{G_{j}}} \frac{\left|N_{G}\left(G_{j}\right)\right|}{|H|}\left(1-\frac{\left|G_{j}^{l^{-1}} \cap H\right|}{\left|G_{j}\right|}\right),
$$

where $G_{j}$ is a representative for the conjugacy class $C_{j}$, and $\Omega_{G_{j}}$ is a left transversal of the normalizer $N_{G}\left(G_{j}\right)$ of $G_{j}$ in $G$.

Proof. Consider the cover $\pi^{H}: S / H \rightarrow S / G$. We will prove the proposition by computing the terms appearing in its corresponding Riemann-Hurwitz formula:

$$
g_{S / H}=[G: H](\gamma-1)+1+\frac{b}{2}
$$

where the ramification contribution $b$ is obtained by considering the three coverings involved. Namely, $\pi_{G}: S \rightarrow S / G, \pi_{H}: S \rightarrow S / H$, and $\pi^{H}: S / H \rightarrow S / G$. First, we associate to any point of $S$ its stabilizer for the total covering $\pi_{G}$. Using the geometric signature and Lemma 3.1, we observe that for every branch value of $C_{j}$-type we have $\left[N_{G}\left(G_{j}\right): G_{j}\right]$ points on $S$ with stabilizer $G_{j}^{l^{-1}}$ (with $G_{j}$ and $l$ as in the Proposition). For each one of these points, the stabilizer for the $H$-action has order $\left|G_{j}^{l^{-1}} \cap H\right|$, and the branch points for the covering $\pi^{H}$ are precisely those points for which this order is different from one. Therefore, for any branch value of $\pi_{G}$ of type $G_{j}$ and each $l \in \Omega_{G_{j}}$, we have

$$
\frac{\left[N_{G}\left(G_{j}\right): G_{j}\right]}{\left|\mathbb{O}_{p}^{H}\right|}=\frac{\left[N_{G}\left(G_{j}\right): G_{j}\right] \cdot\left|G_{j}^{l^{-1}} \cap H\right|}{|H|}
$$

points with ramification index $\left(\left|G_{j}^{l^{-1}}\right| /\left|G_{j}^{l^{-1}} \cap H\right|\right)-1$. Thus, the contribution $b$ from the ramification divisor is

$$
b=\frac{1}{2} \sum_{j=1}^{t} \sum_{l \in \Omega_{G_{j}}} \frac{\left[N_{G}\left(G_{j}\right): G_{j}\right] \cdot\left|G_{j}^{l^{-1}} \cap H\right|}{|H|}\left(\frac{\left|G_{j}^{l^{-1}}\right|}{\left|G_{j}^{l^{-1}} \cap H\right|}-1\right) .
$$


Proposition 3.2 and its proof will prove useful in computing data about the intermediate coverings beyond the genus, such as type of branch values and cycle structure. The following Lemma allows us to rewrite Equation 3.1, as in [8].

Lemma 3.3. Let $G$ be a finite group having subgroups $H$ and $K$, and let $H \backslash G / K$ be the corresponding set of double cosets. Then its cardinality is given by

$$
|H \backslash G / K|=\sum_{l_{j} \in \Omega_{K}} \frac{\left[N_{G}(K): K\right] \cdot\left|K^{l_{j}^{-1}} \cap H\right|}{|H|},
$$

where $\Omega_{K}$ is a left transversal of $N_{G}(K)$ in $G$.

Proof. Set $n=|H \backslash G / K|$ and consider the action of $H$ on the left cosets $I_{K}$ of $K$ in $G$ given by multiplication on the left. Then the stabilizer of $g_{i} \in I_{K}$ in $H$ is $K^{g_{i}^{-1}} \cap H$, and therefore the cardinality of the orbit of $g_{i}$ under $H$ is $\left[H: K^{g_{i}^{-1}} \cap H\right]$.

Let $k=\left|I_{K} / H\right|$ be the number of different orbits under the action of $H$ on $I_{K}$. Then

$$
[G: K]=\left|I_{K}\right|=\sum_{i=1}^{k}\left|\mathbb{O}^{H}\left(g_{i} K\right)\right|=\sum_{i=1}^{k}\left[H: K^{g_{i}^{-1}} \cap H\right] .
$$

Hence $k=n$; that is, $\left|I_{K} / H\right|=|H \backslash G / K|$.

On the other hand, consider the action of $G$ on $I_{K}$ given by multiplication on the left. Then the stabilizer of $g_{i} \in I_{K}$ in $G$ is $K^{g_{i}^{-1}}$. Let us associate to each point $g_{i} \in I_{K}$ its stabilizer in $G$. Then we have the set $I_{K}$ divided into $\left[G: N_{G}(K)\right]$ packages, and each package has $\left[N_{G}(K): K\right]$ points associated to the same stabilizer. Consider now the action restricted to $H \leq G$. Then the stabilizer in $H$ of an element $g_{i} \in I_{K}$ is $K^{g_{i}^{-1}} \cap H$. Therefore, the cardinality of the orbit $\left|\mathbb{O}^{H}\left(g_{i} K\right)\right|$ is $|H| /\left|K^{g_{i}^{-1}} \cap H\right|$. Considering the action of $H$, for each package of points with the same stabilizer in $G$ we will have

$$
\frac{\text { number of points in the package }}{\text { cardinality of the orbit }}=\frac{\left[N_{G}(K): K\right] \cdot\left|K^{g_{i}^{-1}} \cap H\right|}{|H|}
$$

points in $I_{K} / H$.

Taking all the representatives of left cosets of $N_{G}(K)$, we obtain all the packages of points in $I_{K}$, and therefore the cardinality of $I_{K} / H$.

Combining this lemma with Equation 3.1 we obtain a result in [8] that will be used in the proof of Theorem 5.10. 
Corollary 3.4. In the notation of Proposition 3.2, we have, for any subgroup $H \leq G$,

$$
g_{S / H}=[G: H](\gamma-1)+1+\frac{1}{2} \sum_{j=1}^{t}\left([G: H]-\left|H \backslash G / G_{j}\right|\right) .
$$

As the cover $\pi_{H}$ is Galois, the description of any branch value is done by marking it with a number: the order of the stabilizer of any point in its fiber. We will also mark other special points in $S / H$ : the points which are not branch values in $S / H$ (for the cover $S \rightarrow S / H$ ) but which project to a branch value of the cover $S \rightarrow S / G$ will be marked with a one. These points, together with the branch values, will be called marked points for the cover $S \rightarrow S / H$.

As in Proposition 3.2, for each $j$ choose any element $l_{1} \in \Omega_{G_{j}}$ and build the set

$$
L_{1}^{j}:=\left\{l \in \Omega_{G_{j}}:\left|G_{j}^{l^{-1}} \cap H\right|=\left|G_{j}^{l_{1}^{-1}} \cap H\right|\right\} .
$$

If $L_{1}^{j} \subsetneq \Omega_{G_{j}}$, choose any $l_{2} \in \Omega_{G_{j}} \backslash L_{1}^{j}$ and form the corresponding set $L_{2}^{j}$ as before; and so on. Obviously this is a finite algorithmic process. If we call $\nu_{j}$ the number of sets $L_{k}^{j}$ obtained in this way, we have $\sum_{k=1}^{\nu_{j}}\left|L_{k}^{j}\right|=$ $\left[G: N_{G}\left(G_{j}\right)\right]$. With the above notation, we can describe the marked points for the cover $S \rightarrow S / H$ as follows.

Proposition 3.5. Let $S$ be a Riemann Surface with $G$-action and geometric signature $\left(\gamma ;\left[m_{1}, C_{1}\right], \ldots,\left[m_{t}, C_{t}\right]\right)$. Then, there are

$$
c_{k}^{j}:=\left|L_{k}^{j}\right| \cdot\left(\frac{\left[N_{G}\left(G_{j}\right): G_{j}\right] \cdot\left|G_{j}^{l_{k}^{-1}} \cap H\right|}{|H|}\right) \quad\left(1 \leq j \leq t, 1 \leq k \leq \nu_{j}\right)
$$

points on $S / H$ marked with the number $\left|G_{j}^{l_{k}^{-1}} \cap H\right|$ for the action of $H \leq G$.

\subsection{Cycle structure for the cover $\pi^{H}: S / H \rightarrow S / G$}

Proposition 3.6. Let $S$ be a Riemann Surface with $G$-action and geometric signature $\left(\gamma ;\left[m_{1}, C_{1}\right], \ldots,\left[m_{t}, C_{t}\right]\right)$, and let $c_{k}^{j}$ be as in Proposition 3.5. Let $q \in S / G$ be a branch value of type $C_{j}$ for the total covering $\pi_{G}: S \rightarrow S / G$. Then the cycle structure of $\pi^{H}: S / H \rightarrow S / G$ over $q$ is given by an $N_{j}$-tuple, where $N_{j}=\sum_{k=1}^{\nu_{j}} c_{k}^{j}$, which is of the form

$$
(\ldots, \underbrace{\frac{\left|G_{j}\right|}{\left|G_{j}^{l_{k}^{-1}} \cap H\right|}, \cdots, \frac{\left|G_{j}\right|}{\left|G_{j}^{l_{k}^{-1}} \cap H\right|}}_{c_{k}^{j}-\text { times }}, \ldots) .
$$

Proof. This follows directly from Propositions 3.2 and 3.5. 
Propositions 3.2, 3.5 and 3.6 can be implemented algorithmically, which we did using the software G.A.P. [6]. We do not include the routines here, but they can be found in [12]. These propositions show that the geometric signature determines the geometric structure for the lattice of intermediate quotients under the action of $H \leq G$, for all subgroups $H \leq G$. The converse is also true, as we show next.

Theorem 3.7. Let $S$ be a Riemann surface with $G$-action. Then there is a bijective correspondence between geometric structures for the lattice of the subgroups of $G$ and geometric signatures for the action of $G$.

Proof. As we have seen in Propositions 3.2, 3.5 and 3.6, if we know the geometric signature then we know the signature for $\pi_{H}: S \rightarrow S / H$ and cycle structure for $\pi^{H}: S / H \rightarrow S / G$ for any subgroup $H$ of $G$. That is, we know the geometric structure for this lattice.

Conversely, to have $G$ acting on $S$ with two different geometric signatures means that for at least one cyclic subgroup $G_{j}$, the number of branch values of type $G_{j}$ is different. If we take the quotient of $S$ by this $G_{j}$, the quotient projection will have different branching data in both cases. In fact, the genus of $S / G_{j}$ will be different in the two given cases.

\section{Existence of a Riemann surface with $G$-action with a given geometric signature}

In this section we prove a result which allows us to assure not only the existence of a Riemann surface with the action of a given (finite) group, but at the same time allows control on the behaviour of the intermediate quotients and on the dimensions of the subvarieties appearing in the decomposition of its Jacobian, among other things. This is a subtle difference (but important in our setting) with the usual Riemann's existence theorem (see [2, Prop. 2.1, p. 239]) and with the work of Singerman (see [15, Thm. 1, p. 320]). We thank the referee for pointing us the relation, of which we were not aware, between our approach and Singerman's. Examples 4.2 and 4.3 illustrate the difference, which relies mainly on point iii) of Theorem 4.1.

Following ([2, Def. 2.2, p. 239]), we call a $(2 \gamma+t)$-tuple

$$
\left(a_{1}, \ldots, a_{\gamma}, b_{1}, \ldots, b_{\gamma}, c_{1}, \ldots, c_{t}\right)
$$

of elements of $G$ a generating vector of type $\left(\gamma ; m_{1}, \ldots, m_{t}\right)$ if the following conditions are satisfied:

i) $G$ is generated by the elements $\left\{a_{1}, \ldots, a_{\gamma}, b_{1}, \ldots, b_{\gamma}, c_{1}, \ldots, c_{t}\right\}$;

ii) $\operatorname{order}\left(c_{j}\right)=m_{j}$;

iii) $\prod_{i=1}^{\gamma}\left[a_{i}, b_{i}\right] \prod_{j=1}^{t} c_{j}=1$, where $\left[a_{i}, b_{i}\right]=\left(a_{i} \cdot b_{i} \cdot a_{i}^{-1} \cdot b_{i}^{-1}\right)$. 
Theorem 4.1. Given a finite group $G$, there is a compact Riemann surface $S$ of genus $g$ on which $G$ acts with geometric signature $\left(\gamma ;\left[m_{1}, C_{1}\right], \ldots,\left[m_{t}, C_{t}\right]\right)$ if and only if the following three conditions hold.

i) (Riemann-Hurwitz)

$$
g=|G|(\gamma-1)+1+\frac{|G|}{2} \sum_{j=1}^{t}\left(1-\frac{1}{m_{j}}\right) .
$$

ii) The group $G$ has a generating vector $\left(a_{1}, b_{1}, \ldots, a_{\gamma}, b_{\gamma}, c_{1}, \ldots, c_{t}\right)$ of type $\left(\gamma ; m_{1}, \ldots, m_{t}\right)$.

iii) The elements $c_{1}, \ldots, c_{t}$ of the generating vector are such that the subgroup generated by $c_{j}$ is in the conjugacy class $C_{j}, j=1, \ldots, t$.

Proof. Let us suppose first that there is a compact Riemann surface $S$ of genus $g$, where $G$ acts with signature $\left(\gamma ; m_{1}, \ldots, m_{t}\right)$. Condition $(i)$ is clearly satisfied. In order to prove conditions $(i i)$ and $(i i i)$ we need to find a generating vector for $G$ of the desired type.

Consider the unit disc $\Delta=\{z \in \mathbb{C}:|z|<1\}$, the uniformization theorem ([5], [7]) gives us the existence of a discrete torsion-free subgroup $\Gamma^{*}$ of $\operatorname{Aut}(\Delta)$ such that $S=\Delta / \Gamma^{*}$ and $\operatorname{Aut}(S) \cong N\left(\Gamma^{*}\right) / \Gamma^{*}$, where $N\left(\Gamma^{*}\right)$ is the normalizer of $\Gamma^{*}$ in $\operatorname{Aut}(\Delta)$. As $G$ acts on $S$, there is a Fuchsian subgroup $\Gamma$ of $N\left(\Gamma^{*}\right)$ containing $\Gamma^{*}$ as a normal subgroup and such that $G \cong \Gamma / \Gamma^{*}$.

Considering the natural isomorphism between $\Delta / \Gamma$ and $S / G$, we have the following diagram:

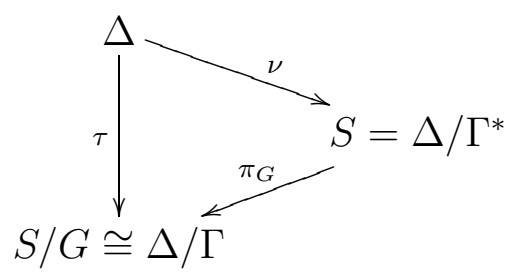

where all of the covers are Galois.

In our situation, the geometric signature $\left(\gamma ;\left[m_{1}, C_{1}\right], \ldots,\left[m_{t}, C_{t}\right]\right)$ allows us ([5, Theorem IV.9.12., p. 234]) to conclude that there are elements $\alpha_{1}, \beta_{1}, \ldots, \alpha_{\gamma}, \beta_{\gamma}, \delta_{1}, \ldots, \delta_{t}$ of $\operatorname{Aut}(\Delta)$ which generate the group $\Gamma$ which as the presentation

$$
\Gamma=<\alpha_{1}, \beta_{1}, \ldots, \alpha_{\gamma}, \beta_{\gamma}, \delta_{1}, \ldots, \delta_{t}: \delta_{1}^{m_{1}}, \ldots, \delta_{t}^{m_{t}}, \prod_{i=1}^{\gamma}\left[\alpha_{i}, \beta_{i}\right] \prod_{j=1}^{t} \delta_{j}>.
$$

Here we have written $(t+1)$ relations after the colon. Furthermore, the covering $\tau: \Delta \rightarrow \Delta / \Gamma=S / G$ has branch values in a finite set $\left\{q_{j}, j=1, \ldots, t\right\}$ 
and its ramification index is $m_{j}$ for each $j$. Moreover, according to $([1, \S 3.2$, p. 8]), the stabilizers of the points in the fiber $\tau^{-1}\left(q_{j}\right)$ are the subgroups in the conjugacy class of $\left\langle\delta_{j}>\right.$.

Let $\theta$ be the isomorphism between $\Gamma / \Gamma^{*}$ and $G$. A generating vector of type $\left(\gamma ; m_{1}, \ldots, m_{t}\right)$ for $G$ consist of the images under $\theta$ of the generating elements for $\Gamma \bmod \Gamma^{*}$ :

$$
\left(\theta\left(\left[\alpha_{1}\right]_{\Gamma^{*}}\right), \theta\left(\left[\beta_{1}\right]_{\Gamma^{*}}\right), \ldots, \theta\left(\left[\alpha_{g}\right]_{\Gamma^{*}}\right), \theta\left(\left[\beta_{g}\right]_{\Gamma^{*}}\right), \theta\left(\left[\delta_{1}\right]_{\Gamma^{*}}\right), \ldots, \theta\left(\left[\delta_{t}\right]_{\Gamma^{*}}\right)\right)
$$

It remains to show that $\theta\left(\left[\delta_{j}\right]_{\Gamma^{*}}\right) \in C_{j}$. Equivalently, we will show that any branch value $q_{j}$ is of type $\theta\left(\left[\delta_{j}\right]_{\Gamma^{*}}\right)$ for $j=1, \ldots, t$.

Consider $q_{j}$ a branch value on $S / G$ and a point $u$ in its fiber under $\tau$. Without loss of generality we may assume that the stabilizer of $u$ is $<\delta_{j}>\leq \Gamma$. Using the fact that the group acting on $\Delta / \Gamma^{*}$ is $\Gamma / \Gamma^{*}$, we have that $\nu(u) \in$ $\pi_{G}^{-1}\left(q_{j}\right)$ with stabilizer $\left\langle\delta_{j}>\Gamma^{*} / \Gamma^{*} \leq \Gamma / \Gamma^{*}\right.$, which corresponds to the subgroup $<\theta\left(\left[\delta_{j}\right]_{\Gamma^{*}}\right)>$.

Conversely, suppose there is a tuple of generating vectors $\left(a_{1}, b_{1}, \ldots, a_{\gamma}, b_{\gamma}, c_{1}, \ldots, c_{t}\right)$ for $G$. Let $Y$ be a compact Riemann surface of genus $\gamma$, set $B=\left\{q_{1}, \ldots, q_{t}\right\} \subset Y$ and let $q \in Y \backslash B$ be a base point. Then the fundamental group for $Y \backslash B$ has a presentation of the form

$$
\pi_{1}(Y \backslash B, q)=<\alpha_{1}, \beta_{1}, \ldots, \alpha_{\gamma}, \beta_{\gamma}, \delta_{1}, \ldots, \delta_{t}: \prod_{i=1}^{\gamma}\left[\alpha_{i}, \beta_{i}\right] \prod_{j=1}^{t} \delta_{j}>
$$

Let $\phi: G \rightarrow S_{|G|}$ be the permutation representation of $G$ and define the group homomorphism $\rho: \Pi_{1}(Y \backslash B, q) \rightarrow S_{|G|}$ by $\alpha_{i} \mapsto \phi\left(a_{i}\right), \beta_{i} \mapsto \phi\left(b_{i}\right)$, for $i=1, \ldots, \gamma$ and $\delta_{j} \mapsto \phi\left(c_{j}\right)$, for $j=1, \ldots, t$.

The image of $\rho$ is $\phi(G)$, which is a transitive subgroup of $S_{|G|}$. Therefore, there is an associated branched covering $f: S \rightarrow Y$ of degree $|G|$ and branch values in $B$. According to [10], this covering corresponds to the subgroup

$$
H=\left\{[\gamma] \in \Pi_{1}(Y \backslash B, q): \rho([\gamma]) \in S_{|G|-1} \cap \phi(G)\right\} \cong \operatorname{ker}(\rho) \text {. }
$$

Hence the Galois group of the regular covering $f: S \rightarrow Y$ is

$$
\operatorname{Gal}(f: S \rightarrow Y) \cong \frac{\pi_{1}(Y \backslash B, q)}{f_{*}\left(\pi_{1}\left(S \backslash f^{-1}(B), p\right)\right)}=\frac{\pi_{1}(Y \backslash B, q)}{\operatorname{ker}(\rho)} \cong \operatorname{Im} \rho \cong G .
$$

Thus $G$ acts on $S$ and $S / G \cong Y$. Furthermore, as the Riemann-Hurwitz formula holds, the genus of $S$ is $g$ and the marked points are $\left\{q_{1}, \ldots, q_{t}\right\}$. Considering the natural homomorphism between the fundamental group of $S / G$ and $G$, we see that the type of $q_{j}$ is $\phi\left(c_{j}\right)$. 
Example 4.2. Consider the dihedral group $D_{4}=<x, y: x^{4}, y^{2},(x y)^{2}>$. There is a Riemann surface with $D_{4}$-action with signature $(0 ; 4,2,2)$. In fact, it is the Riemann sphere, where $D_{4}$ acts with geometric signature $(0 ;[4, \bar{x}],[2, \bar{y}],[2, \overline{x y}])$. But there is no Riemann surface where $D_{4}$ acts and geometric signature $\left(0 ;[4, \bar{x}],\left[2, \overline{x^{2}}\right],\left[2, \overline{x^{2}}\right]\right)$, because we cannot find a generating vector of $D_{4}$ whose elements belong to these conjugacy classes.

Example 4.3. Consider the Weyl group $W C_{3}$ of type $C_{3}$ [17], isomorphic to $\mathbb{Z}_{2}^{3} \rtimes \mathcal{S}_{3}$, where $\mathcal{S}_{3}=<a, b: a^{3}, b^{2},(a b)^{2}>$. We denote by $x, y, z$ generators of $\mathbb{Z}_{2}^{3}$. Using Theorem 4.1, we can prove that there are two actions of $G$ on genus 3 surfaces, with different geometric signatures given

$$
\left(0 ;\left[\overline{G_{5}}, 6\right],\left[\overline{G_{3}}, 4\right],\left[\overline{G_{1}}, 2\right]\right) \quad \text { and }\left(0 ;\left[\overline{G_{5}}, 6\right],\left[\overline{G_{4}}, 4\right],\left[\overline{G_{2}}, 2\right]\right)
$$

where the (different) conjugacy classes $\overline{G_{j}}$ are given by the following representatives:

\begin{tabular}{|l||l|l|l|l|l|}
\hline $\begin{array}{l}\text { Repre- } \\
\text { sentative }\end{array}$ & $\begin{array}{l}G_{1}= \\
<x y z b>\end{array}$ & $\begin{array}{l}G_{2}= \\
<y z a b>\end{array}$ & $\begin{array}{l}G_{3}= \\
<x y a b>\end{array}$ & $\begin{array}{l}G_{4}= \\
<y a b>\end{array}$ & $\begin{array}{l}G_{5}= \\
<x a^{2}>\end{array}$ \\
\hline Order & 2 & 2 & 4 & 4 & 6 \\
\hline
\end{tabular}

First, we verify that condition $(i)$ of the Theorem holds. Then, we find a generating vector verifying conditions $(i i)$ and $(i i i)$ for each case as follows, $c_{5}=x a^{2}, c_{3}=x y a b$ and $c_{1}=x y z b$, for the first one; and $d_{5}=x a^{2}, d_{4}=z a b$ and $d_{2}=b$, for the second one.

According to ([2, p. 255]), these two actions are topologically equivalent. Nevertheless, we see from Section 3 that since they have different geometric signatures, they also have different geometric structures for the lattice of intermediate quotients. In fact, consider $H_{1}=<y, z, x y z a b>$ and $H_{2}=<y, z, a b>$, two non-conjugate subgroups of order 8 , both isomorphic to $D_{4}$. Using Section 3, we can describe the genus of the intermediate quotients of $S$ by $H_{1}$ and $H_{2}$. In the first case, the genus of the quotient by $H_{1}$ is 0 and by $H_{2}$ is 1 . In the second case the genus of the quotient by $H_{1}$ is 1 and by $H_{2}$ is 0 .

As we will show on Section 5, this difference is also reflected in the isotypical decomposition of the rational representation for the action of $G$ on the Jacobian variety $J S$ of $S$.

In this case, we can find the affine equation for the surface with these $G$-actions, $S: y^{2}=x^{8}+14 x^{4}+1$. The two actions presented arise from the existence of two different injections from the group $W C_{3}$ to $\operatorname{Aut}(S)$. We can summarize them as follows: 


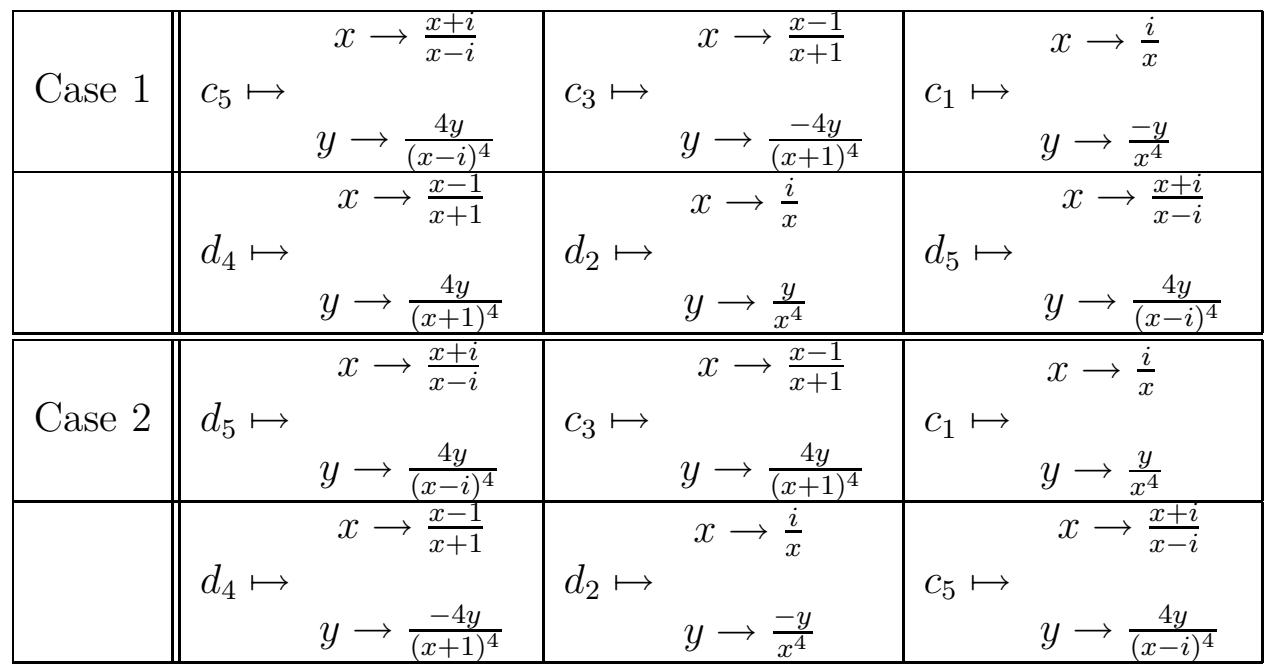

Remark 4.4. This is an useful example, although it is not new. As Wolfart pointed in [20], it plays an important role in several respects, he proved in [19] (theorem 5, p. 116), that it is the only exception to the fact that compact Riemann surface of genus 3 with many automorphisms have Jacobians of CM type. The same is pointed in [16].

\section{Isotypical decomposition for the rational representa- tion}

We start by giving some results which follow directly from the representation theory in [4], [8] and [14]. Let $U \in \operatorname{Irr}_{\mathbb{C}}(G)$ be a complex irreducible representation. We recall that $\ell_{U}$ is the Schur index of $U$, and $K_{U}$ is a field extension of $\mathbb{Q}$ (see Section 2). We call the set $\left\{U^{\sigma}: \sigma \in \operatorname{Gal}\left(K_{U} / \mathbb{Q}\right)\right\}$ the Galois class of $U$.

Theorem 5.1. ([4, §70, p. 479]) Let $\left\{U_{1}, \ldots, U_{r}\right\}$ be a full set of representatives of Galois classes from the set $\operatorname{Irr}_{\mathbb{C}}(G)$ and let $K_{j}=K_{U_{j}}$. Then for each rational irreducible representation $\mathcal{W}$ of $G$ there exists precisely one $U_{j}$ satisfying

$$
\mathcal{W} \otimes_{\mathbb{Q}} \mathbb{C} \cong \bigoplus_{i=1}^{\ell_{j}} \bigoplus_{\sigma \in \operatorname{Gal}\left(K_{j} / \mathbb{Q}\right)} U_{j}^{\sigma}=:\left(\bigoplus_{\sigma \in \operatorname{Gal}\left(K_{j} / \mathbb{Q}\right)} U_{j}^{\sigma}\right)^{\ell_{j}} .
$$

Conversely, the right-hand side of (5.1) is the complexification of a rational irreducible representation of $G$ for each $U_{j}$.

Lemma 5.2. ([4], [14]) Let $\rho: G \rightarrow G L(U)$ be a complex irreducible representation. Then $\operatorname{dim}\left(\operatorname{Fix}_{G}\left(U \otimes U^{*}\right)\right)=1$. 
Corollary 5.3. ([4], [14]) Let $\theta: G \rightarrow G L(V)$ be a complex representation of the group $G$ with isotypical decomposition

$$
V=U_{1}^{n_{1}} \oplus \ldots \oplus U_{s}^{n_{s}}
$$

with $\rho_{j}: G \rightarrow G L\left(U_{j}\right)$. Then:

1. $\operatorname{dim}\left(\operatorname{Fix}_{G}\left(V \otimes U_{j}^{*}\right)\right)=n_{j}$ for $j=1, \ldots, s$.

2. The representation of $G$ on the space $\operatorname{Fix}_{G}\left(V \otimes U_{j}^{*}\right)$ is $n_{j} \rho_{0}$, with $\rho_{0}$ the trivial one-dimensional representation of $G$.

3. The isotypical component $U_{j}^{n_{j}}$ is isomorphic as a G-module to $U_{j} \otimes \operatorname{Fix}_{G}\left(V \otimes U_{j}^{*}\right)$.

Corollary 5.3 combined with Theorem 5.1, give the following decomposition of any complex representation.

Theorem 5.4. (cf. [8]) Given a complex representation $\rho: G \rightarrow G L(V)$, we can write the isotypical decomposition for $V$ as follows:

$$
V \cong \bigoplus_{U \in \operatorname{Irr}_{\mathbb{C}} G} U \otimes \operatorname{Fix}_{G}\left(V \otimes U^{*}\right)
$$

We use Theorems 5.4 and 5.1 to write the isotypical decomposition for the complexification of any rational representation of $G$ in the following way.

Proposition 5.5. Let $G$ be a finite group and consider $\left\{U_{1}, \ldots, U_{r}\right\}$, a set constructed by taking one representative from each Galois class of all the complex irreducible representations of $G$. Then every rational representation $\mathcal{W}$ of $G$ can be written as

$$
\mathcal{W} \otimes \mathbb{C} \cong \bigoplus_{j=1}^{r}\left(\bigoplus_{\sigma} U_{j}^{\sigma}\right) \otimes V_{j}
$$

where

$$
K_{j}=\mathbb{Q}\left(\chi_{U_{j}}(g): g \in G\right), \quad V_{j}=\operatorname{Fix}_{G}\left(\mathcal{W} \otimes U_{j}^{*}\right)
$$

and $\sigma$ runs over $\operatorname{Gal}\left(K_{j} / \mathbb{Q}\right)$.

Proof. Just decompose the direct sum from Theorem 5.4 using the Galois classes.

When the group $G$ acts on a Riemann Surface $S$, there is a naturally associated rational representation $\rho_{\mathbb{Q}}: G \rightarrow G L\left(H_{1}(S, \mathbb{Z}) \otimes \mathbb{Q}\right)$. We want to find the dimension of each complex irreducible representation in the complexification of $\rho_{\mathbb{Q}}$. 
Applying Proposition 5.5 to $\rho_{\mathbb{Q}}$ we obtain

Corollary 5.6. Let $S$ be a Riemann surface with G-action. Consider a full set of representatives $\left\{U_{1}, \ldots, U_{r}\right\}$ of the different Galois classes of complex irreducible representations of $G$, and the rational representation $\rho_{\mathbb{Q}}$ for the action of $G$ on the corresponding Jacobian variety. Then

$$
\rho_{\mathbb{Q}} \otimes \mathbb{C} \cong \bigoplus_{j=1}^{r}\left(\bigoplus_{\sigma} U_{j}^{\sigma}\right) \otimes V_{j}
$$

where $K_{j}=\mathbb{Q}\left(\chi_{U_{j}}(g): g \in G\right), V_{j}=\operatorname{Fix}_{G}\left(\rho_{\mathbb{Q}} \otimes U_{j}^{*}\right)$ and $\sigma$ runs over $\operatorname{Gal}\left(K_{j} / \mathbb{Q}\right)$.

The multiplicities we are looking for are precisely the complex dimensions of the vector spaces $V_{j}$. We will use the information concerning the intermediate coverings to find these dimensions.

Proposition 5.7. In the notation of Corollary 5.6, for each subgroup $H \leq G$ we have

$$
\operatorname{dim}\left(\operatorname{Fix}_{H}\left(\rho_{\mathbb{Q}} \otimes \mathbb{C}\right)\right)=\sum_{j=1}^{r} \sum_{\sigma \in \operatorname{Gal}\left(K_{j} / \mathbb{Q}\right)} \operatorname{dim}\left(\operatorname{Fix}_{H}\left(U_{j}^{\sigma}\right)\right) \cdot \operatorname{dim}\left(V_{j}\right) .
$$

Proof. For any vector space $V$ having a $G$-equivariant decomposition $V=$ $U \oplus W$, we have $\operatorname{Fix}_{H}(V)=\operatorname{Fix}_{H}(U) \oplus \operatorname{Fix}_{H}(W)$, for all $H \leq G \leq G L(V)$. Using $\operatorname{Fix}_{H}\left(V_{j}\right)=V_{j}$, we obtain from the decomposition given by Corollary 5.6 that $\operatorname{Fix}_{H}\left(\rho_{\mathbb{Q}} \otimes \mathbb{C}\right) \cong \bigoplus_{j=1}^{r} \operatorname{Fix}_{H}\left(\bigoplus_{\sigma} U_{j}^{\sigma}\right) \otimes V_{j}$. Comparing dimensions, we obtain $(5.2)$.

Since $\operatorname{dim}\left(\operatorname{Fix}_{H}\left(\rho_{\mathbb{Q}} \otimes \mathbb{C}\right)\right)=2 g_{S / H}$ for all $H \leq G([5, \S V .2 .2]),.(5.2)$ gives a square system of linear equations when $H$ runs over the set $\left\{H_{1}, \ldots H_{r}\right\}$ of all cyclic subgroups of $G$ up to conjugacy. The $r$ unknowns are $\operatorname{dim}\left(V_{j}\right)$. To solve this system is equivalent to find the multiplicity of each complex irreducible representation in the isotypical decomposition of $\rho_{\mathbb{Q}} \otimes \mathbb{C}$.

The system for the "unknowns" $\operatorname{dim}\left(V_{i}\right)$ is:

$$
2 g_{S / H_{j}}=\sum_{i=1}^{r} \operatorname{dim}\left(V_{i}\right) \cdot \sum_{\sigma \in \operatorname{Gal}\left(K_{i} / \mathbb{Q}\right)} \operatorname{dim}\left(\operatorname{Fix}_{H_{j}}\left(U_{i}^{\sigma}\right)\right) \quad(j=1, \ldots, r) .
$$

Our next step will be to show that the system 5.3 admits a unique solution. We will prove this in Lemma 5.8 , by showing the invertibility of the $r \times r$ matrix $\Omega=\left(a_{i j}\right)$, where

$$
a_{i j}:=\sum_{\sigma \in \operatorname{Gal}\left(K_{i} / \mathbb{Q}\right)} \operatorname{dim}\left(\operatorname{Fix}_{H_{j}}\left(U_{i}^{\sigma}\right)\right) .
$$

After this, we will write down the solution in Theorem 5.10. 
Lemma 5.8. The matrix $\Omega$ defined above is invertible.

Proof. Consider the complex character table of $G$ arranged in the following way: the rows are indexed by representatives $c_{i}$ of the conjugacy classes of elements of $G$ arranged by increasing order $\left(\left|c_{i}\right| \leq\left|c_{i+1}\right|\right)$, the columns are indexed by the complex irreducible characters $\chi_{i}$ arranged in packages of complete Galois classes. The coefficients of the table are, as usual, the value of the character on the representative. To simplify notation, we consider $\sigma$ as being in the appropriate Galois group. The table looks as follows:

\begin{tabular}{c|cccccc} 
& $\chi_{1}$ & $\chi_{1}^{\sigma}$ & $\ldots$ & $\chi_{j}$ & $\chi_{j}^{\sigma}$ & $\ldots$ \\
\hline$c_{1}$ & $\chi_{1}\left(c_{1}\right)$ & $\chi_{1}^{\sigma}\left(c_{1}\right)$ & $\ldots$ & $\chi_{j}\left(c_{1}\right)$ & $\chi_{j}^{\sigma}\left(c_{1}\right)$ & $\ldots$ \\
$\vdots$ & $\vdots$ & $\vdots$ & $\vdots$ & $\vdots$ & $\vdots$ & $\vdots$ \\
$c_{s}$ & $\chi_{1}\left(c_{s}\right)$ & $\chi_{1}^{\sigma}\left(c_{s}\right)$ & $\ldots$ & $\chi_{j}\left(c_{s}\right)$ & $\chi_{j}^{\sigma}\left(c_{s}\right)$ & $\ldots$
\end{tabular}

which defines an invertible $s \times s$ matrix $A$, due to the orthogonality relations of characters. We will use this fact to show that $\Omega$ is also invertible.

Let $B$ be the $s \times r$ matrix resulting from adding the columns of $A$ associated to representations of the same Galois class. This matrix $B$ has maximal rank $r$, where $r$ is the number of cyclic subgroups of $G$ up to conjugacy. Call $\theta_{j}$ the following class function

$$
\theta_{j}:=\sum_{\sigma \in \operatorname{Gal}\left(K_{j}: \mathbb{Q}\right)} \chi_{j}^{\sigma} .
$$

We can write $B$ as follows:

\begin{tabular}{c|ccccc} 
& $\theta_{1}$ & $\ldots$ & $\theta_{j}$ & $\ldots$ & $\theta_{r}$ \\
\hline$c_{1}$ & $\theta_{1}\left(c_{1}\right)$ & $\ldots$ & $\theta_{j}\left(c_{1}\right)$ & $\ldots$ & $\theta_{r}\left(c_{1}\right)$ \\
$\vdots$ & $\vdots$ & $\ddots$ & $\vdots$ & $\ddots$ & $\vdots$ \\
$c_{s}$ & $\theta_{1}\left(c_{s}\right)$ & $\ldots$ & $\theta_{j}\left(c_{s}\right)$ & $\ldots$ & $\theta_{r}\left(c_{s}\right)$
\end{tabular}

As $\theta_{j}\left(c_{i}\right)=\theta_{j}\left(c_{k}\right)$, when $\left\langle c_{i}>\right.$ and $\left\langle c_{k}>\right.$ are conjugate cyclic subgroups of $G$, we erase some rows of $B$ and keep just one row among those corresponding to elements generating conjugate subgroups of $G$. Thus we obtain a new invertible square matrix $B^{\prime}$ of size $r$ :

\begin{tabular}{c|ccccc} 
& $\theta_{1}$ & $\ldots$ & $\theta_{j}$ & $\ldots$ & $\theta_{r}$ \\
\hline$c_{1}$ & $\theta_{1}\left(c_{1}\right)$ & $\ldots$ & $\theta_{j}\left(c_{1}\right)$ & $\ldots$ & $\theta_{r}\left(c_{1}\right)$ \\
$\vdots$ & $\vdots$ & $\ddots$ & $\vdots$ & $\ddots$ & $\vdots$ \\
$c_{r}$ & $\theta_{1}\left(c_{r}\right)$ & $\ldots$ & $\theta_{j}\left(c_{r}\right)$ & $\ldots$ & $\theta_{r}\left(c_{r}\right)$
\end{tabular}

where $c_{i}$ is now a representative of the set we denote by $\left[c_{i}\right]$, consisting of all the elements of the conjugacy class of $c_{i}$ and all the elements of the conjugacy class of $c_{j}$ when $c_{i}$ and $c_{j}$ generate conjugate cyclic subgroups of $G$. 
On the other hand, $\Omega$ is a square matrix of size $r$ with coefficients given by

$$
a_{i j}:=\sum_{\sigma \in \operatorname{Gal}\left(K_{i} / \mathbb{Q}\right)} \operatorname{dim}\left(\operatorname{Fix}_{H_{j}}\left(U_{i}^{\sigma}\right)\right)=\frac{1}{\left|H_{j}\right|} \sum_{\sigma \in \operatorname{Gal}\left(K_{i} / \mathbb{Q}\right)} \sum_{h \in H_{j}} \chi_{i}(h) .
$$

Rearranging the sums and using the notation above, we obtain

$$
a_{i j}=\frac{1}{\left|H_{j}\right|} \sum_{h \in H_{j}} \theta_{i}(h)=\frac{1}{\left|H_{j}\right|} \sum_{k=1}^{r} \theta_{i}\left(c_{k}\right)\left|\left[c_{k}\right] \cap H_{j}\right| .
$$

We also rearrange $\Omega$ using the same order as for $B^{\prime}$. It is clear that every row $i$ of $\Omega$ results from elementary operations applied to the rows $k=1, \ldots, i$ of $B^{\prime}$. As $B^{\prime}$ is invertible, so is $\Omega$.

Lemma 5.9. In the notation of Lemma 3.3, we have

$$
|H \backslash G / K|=\frac{1}{|H|} \sum_{a \in H} \frac{|G| \cdot|K \cap \bar{a}|}{|K| \cdot|\bar{a}|}
$$

where $\bar{a}$ means the conjugacy class of a in $G$.

Proof. Following the proof of Lemma 3.3, the idea is to obtain the cardinality of $I_{K} / H$ in a different way. The cardinality of the orbit of $g_{i} \in I_{K}$ under $a \in H$ is $|\bar{a}| /|\bar{a} \cap K|$. The number of orbits on $I_{K}$ under $a \in H$ is $[G: K] \cdot|\bar{a} \cap K| /|\bar{a}|$. Therefore, the number of $H$-orbits in $I_{K}$ is

$$
\sum_{a \in H} \frac{|G| \cdot|K \cap \bar{a}|}{|K| \cdot|\bar{a}|}
$$

Theorem 5.10. Let $G$ be a finite group acting on a Riemann surface $S$, with geometric signature $\left(\gamma ;\left[m_{1}, C_{1}\right], \ldots,\left[m_{t}, C_{t}\right]\right)$. For each non trivial complex irreducible representation $\theta_{i}: G \rightarrow G L\left(U_{i}\right)$, the multiplicity $n_{i}$ of $\theta_{i}$ in the isotypical decomposition of $\rho_{\mathbb{Q}} \otimes \mathbb{C}$ is given by

$$
n_{i}=2 \operatorname{dim}\left(U_{i}\right)(\gamma-1)+\sum_{k=1}^{t}\left(\operatorname{dim}\left(U_{i}\right)-\operatorname{dim}\left(\operatorname{Fix}_{G_{k}}\left(U_{i}\right)\right)\right)
$$

where $G_{k}$ is a representative of the conjugacy class $C_{k}$.

Proof. As we noted before Proposition 5.7, the multiplicity $n_{i}$ of each complex irreducible representation in the isotypical decomposition of $\rho_{\mathbb{Q}}$, corresponds to the factor $\operatorname{dim}\left(V_{i}\right)$ in (5.3). The idea of the proof is to replace in (5.3) the expression (5.4) for $n_{i}$ and the expression for $g_{S / H_{j}}$ given in Corollary 3.4, and to then verify that we indeed have equality. 
In the following we omit some parentheses in order to simplify the notation.

We write the multiplicity for the trivial complex representation $U_{1}$, which we know is $2 \gamma$, in the same way

$$
n_{1}=\operatorname{dim} V_{1}=2+2 \operatorname{dim} U_{1}(\gamma-1)+\sum_{k=1}^{t}\left(\operatorname{dim} U_{1}-\operatorname{dim} \operatorname{Fix}_{G_{k}} U_{1}\right) .
$$

On the other hand, we rewrite each equation (5.3) producing a new system

$$
2 g_{S / H_{j}}=n_{1} \sum_{\sigma \in \operatorname{Gal}\left(K_{1}: \mathbb{Q}\right)} \operatorname{dim} \operatorname{Fix}_{H_{j}} U_{1}^{\sigma}+\sum_{i=2}^{r} n_{i} \sum_{\sigma} \operatorname{dim} \operatorname{Fix}_{H_{j}} U_{i}^{\sigma}
$$

where $j=1, \ldots, r$, and in the second sum $\sigma$ runs over $\operatorname{Gal}\left(K_{i} / \mathbb{Q}\right)$. Replacing in (5.6) the expressions for $n_{1}$ and $n_{i}(i=2, \ldots, r)$ given in (5.5) and (5.4), we obtain:

$$
\begin{array}{r}
2 g_{S / H_{j}}=\sum_{\sigma} \operatorname{dim} \operatorname{Fix}_{H_{j}} U_{1}^{\sigma}\left(2+2 \operatorname{dim} U_{1}(\gamma-1)+\sum_{k=1}^{t}\left(\operatorname{dim} U_{i}-\operatorname{dim} \operatorname{Fix}_{G_{k}} U_{i}\right)\right) \\
+\sum_{i=2}^{r}\left(\sum_{\sigma} \operatorname{dim}^{t} \operatorname{Fix}_{H_{j}} U_{i}^{\sigma}\right)\left(2 \operatorname{dim} U_{i}(\gamma-1)+\sum_{k=1}^{t}\left(\operatorname{dim} U_{i}-\operatorname{dim} \operatorname{Fix}_{G_{k}} U_{i}\right)\right)
\end{array}
$$

where $\sigma$ runs over the appropriate Galois group. Grouping the term for $U_{1}$ with the sum and simplifying, the system now looks as follows:

$$
=1+\sum_{i=1}^{r} \sum_{\sigma} \operatorname{dim} \operatorname{Fix}_{H_{j}} U_{i}^{\sigma}\left(\operatorname{dim} U_{i}(\gamma-1)+\frac{1}{2} \sum_{k=1}^{t}\left(\operatorname{dim} U_{i}-\operatorname{dim} \operatorname{Fix}_{G_{k}} U_{i}\right)\right) .
$$

We compare, term by term, the expressions for $g_{S / H_{j}}$ given by (5.7) versus the one given by Equation (3.3), taking $H=H_{j}$. The terms corresponding to the factor $(\gamma-1)$ are

$$
\sum_{i=1}^{r} \sum_{\sigma \in \operatorname{Gal}\left(K_{i} / \mathbb{Q}\right)}\left(\operatorname{dim}^{\operatorname{Fix}_{H_{j}}} U_{i}^{\sigma}\right) \operatorname{dim} U_{i} \quad \text { vs. } \quad \frac{|G|}{\left|H_{j}\right|}
$$

For the left term we have

$$
\sum_{i=1}^{r} \sum_{\sigma \in \operatorname{Gal}\left(K_{i} / \mathbb{Q}\right)}\left(\operatorname{dim} \operatorname{Fix}_{H_{j}} U_{i}^{\sigma}\right) \operatorname{dim} U_{i}=\frac{1}{\left|H_{j}\right|} \sum_{h \in H_{j}} \sum_{i=1}^{r} \sum_{\sigma} \chi_{i}^{\sigma}(h) \chi_{i}(\mathrm{id}) .
$$


But $\chi_{i}(\mathrm{id})=\chi_{i}^{\sigma}(\mathrm{id})$ for all $\sigma$ in the corresponding Galois group and for all $i$. Thus, the former is equal to

$$
\frac{1}{\left|H_{j}\right|} \sum_{h \in H_{j}} \chi_{R E G}(h)=\frac{|G|}{\left|H_{j}\right|} .
$$

The terms associated to the geometric signature are

$$
\begin{aligned}
& \sum_{i=1}^{r} \sum_{\sigma \in \operatorname{Gal}\left(K_{i} / \mathbb{Q}\right)}\left(\operatorname{dim} \operatorname{Fix}_{H_{j}} U_{i}^{\sigma}\right)\left(\frac{1}{2} \sum_{k=1}^{t}\left(\operatorname{dim} U_{i}-\operatorname{dim} \operatorname{Fix}_{G_{k}} U_{i}\right)\right) \\
& \text { vs. } \frac{1}{2} \sum_{k=1}^{t}\left(\frac{|G|}{\left|H_{j}\right|}-\left|H_{j} \backslash G / G_{k}\right|\right) .
\end{aligned}
$$

It is then clear that all that remains to prove is the equality

$$
\sum_{i=1}^{r} \sum_{\sigma \in \operatorname{Gal}\left(K_{i} / \mathbb{Q}\right)}\left(\operatorname{dim} \operatorname{Fix}_{H_{j}} U_{i}^{\sigma}\right)\left(\operatorname{dim} \operatorname{Fix}_{G_{k}} U_{i}\right)=\left|H_{j} \backslash G / G_{k}\right| .
$$

The left term may be written as follows

$$
\frac{1}{\left|H_{j}\right| \cdot\left|G_{k}\right|} \sum_{i=1}^{r} \sum_{\sigma \in \operatorname{Gal}\left(K_{i} / \mathbb{Q}\right)}\left(\sum_{a \in H_{j}} \sum_{b \in G_{k}} \overline{\chi_{i}^{\sigma}}(a) \chi_{i}^{\sigma}(b)\right)
$$

using the next two known results [4],

$$
\begin{array}{r}
\frac{|G|}{|\bar{g}|}=\sum_{\chi \in \operatorname{Irr} \mathbb{C} G} \overline{\chi(g)} \chi(g)=\sum_{i=1}^{r} \sum_{\sigma \in \operatorname{Gal}\left(K_{i} / \mathbb{Q}\right)} \overline{\chi_{i}^{\sigma}}(g) \chi_{i}^{\sigma}(g), \\
\sum_{\chi \in \operatorname{Irr}_{\mathbb{C}} G} \overline{\chi\left(g_{1}\right)} \chi\left(g_{2}\right)=\sum_{i=1}^{r} \sum_{\sigma \in \operatorname{Gal}\left(K_{i} / \mathbb{Q}\right)} \overline{\chi_{i}^{\sigma}}\left(g_{1}\right) \chi_{i}^{\sigma}\left(g_{2}\right)=0
\end{array}
$$

if $g_{1}$ is not conjugate to $g_{2}$. Thus, the term of Equation 5.8 vanishes unless $b \in G_{k}$ is conjugate to $a \in H_{j}$. In this case it is equal to $|G| /|\bar{a}|$, and this happens precisely on $\left|G_{k} \cap \bar{a}\right|$ elements of $G_{k}$. Using Lemma 5.9, the Theorem is proved.

Corollary 5.11. Let $G$ be a finite group acting on a Riemann surface $S$ with geometric signature $\left(\gamma ;\left[m_{1}, C_{1}\right], \ldots,\left[m_{t}, C_{t}\right]\right)$. Then for each non trivial rational irreducible representation $\mathcal{W}_{i}$ of $G$, the multiplicity $e_{i}$ of $\mathcal{W}_{i}$ in the isotypical decomposition of $\rho_{\mathbb{Q}}$ is given by

$$
e_{i}=\frac{2 \operatorname{dim}\left(U_{i}\right)(\gamma-1)+\sum_{k=1}^{t}\left(\operatorname{dim} U_{i}-\operatorname{dim}_{\operatorname{Fix}_{G_{k}}} U_{i}\right)}{\ell_{i}},
$$


where $G_{k}$ is a representative of the conjugacy class $C_{k}$, $\operatorname{dim}\left(U_{i}\right)$ is the dimension of the complex irreducible representation associated to $\mathcal{W}_{i}$, and $\ell_{i}$ is the Schur index of $U_{i}$.

We can compute (in terms of the geometric signature) the dimension of each subvariety in the isogeny $G$-equivariant decomposition of the Jacobian variety as follows (cf. Theorem 2.1).

Theorem 5.12. Let $G$ be a finite group acting on a Riemann surface $S$ with geometric signature $\left(\gamma ;\left[m_{1}, C_{1}\right], \ldots,\left[m_{t}, C_{t}\right]\right)$. Then the dimension of any subvariety $B_{i}$ associated to a non trivial rational irreducible representation $\mathcal{W}_{i}$, in the $G$-equivariant isogeny decomposition of the corresponding Jacobian variety $J S$, is given by

$$
\operatorname{dim} B_{i}=k_{i}\left(\operatorname{dim} U_{i}(\gamma-1)+\frac{1}{2} \sum_{k=1}^{t}\left(\operatorname{dim} U_{i}-\operatorname{dim} \operatorname{Fix}_{G_{k}} U_{i}\right)\right)
$$

where $G_{k}$ is a representative of the conjugacy class $C_{k}, \operatorname{dim} U_{i}$ is the dimension of a complex irreducible representation $U_{i}$ associated to $\mathcal{W}_{i}$,

$$
K_{i}=\mathbb{Q}\left(\chi_{U_{i}}(g): g \in G\right),
$$

$\ell_{i}$ is the Schur index of $U_{i}$, and $k_{i}=\ell_{i} \cdot\left|\operatorname{Gal}\left(K_{i}: \mathbb{Q}\right)\right|$.

Proof. Consider the decomposition of $J S$ given in Theorem 2.1

$$
J S \sim B_{1}^{s_{1}} \times \cdots \times B_{r}^{s_{r}}
$$

where $s_{i}=\operatorname{dim}\left(U_{i}\right) / \ell_{i}$. Thus, each $G$-stable factor $B_{i}^{s_{i}}$ has dimension $\operatorname{dim}\left(B_{i}\right) \operatorname{dim}\left(U_{i}\right) / \ell_{i}$. Comparing the dimension of each factor with the isotypical $\mathbb{Q}$-decomposition for $\rho_{\mathbb{Q}}$ given in Corollary 5.11, we have

$$
2 \frac{\operatorname{dim}\left(B_{i}\right) \operatorname{dim}\left(U_{i}\right)}{\ell_{i}}=\operatorname{dim}\left(U_{i}\right) \ell_{i}\left|\operatorname{Gal}\left(K_{i}: \mathbb{Q}\right)\right| e_{i}
$$

where $e_{i}$ is the multiplicity given in Corollary 5.11 .

We observe that even though $B_{i}$ is defined only up to isogeny, its dimension is well defined.

It follows immediately from (5.10) that if $\gamma \geq 2$, then the dimension of each subvariety $B_{i}$ in the $G$-equivariant decomposition of $J S$ is positive, a result already obtained in [9]. If $\gamma=0$, then we know that at least the dimension of $B_{1}$ (corresponding to the trivial representation of $G$ ) is zero.

In our next result we analyze the case $\gamma=1$. 
Corollary 5.13. In the notation of Theorem 5.12, assume that $\gamma=1$. Consider $B_{i}$ a subvariety associated to a non trivial representation $\mathcal{W}_{i}$, and $U_{i}$ a complex irreducible representation associated to $\mathcal{W}_{i}$.

Then the following conditions are equivalent.

1. The dimension of $B_{i}$ is 0 ;

2. $C_{k} \subseteq \operatorname{ker}\left(U_{i}\right)$ for all $k=1, \ldots, t$;

3. the covering $\pi^{\operatorname{ker} U_{i}}: S / \operatorname{ker} U_{i} \rightarrow S / G$ is unramified;

4. the genus of $S / \operatorname{ker} U_{i}$ is 1 .

Moreover, if $\operatorname{dim} B_{i}=0$ then the degree of $U_{i}$ is 1 .

Proof. Suppose $\operatorname{dim}\left(B_{i}\right)=0$. Then from equation (5.10) we obtain that $\operatorname{dim}\left(U_{i}\right)=\operatorname{dim}\left(\operatorname{Fix}_{G_{k}}\left(U_{i}\right)\right)$ for all $G_{k}$.

Therefore $G_{k} \leq \operatorname{ker}\left(U_{i}\right)$ for all $k$. As $\operatorname{ker}\left(U_{i}\right)$ is a normal subgroup of $G$, $G_{k}^{l} \leq \operatorname{ker}\left(U_{i}\right)$ for all $l \in G$, and we obtain (2). Furthermore, the ramification divisors for the coverings $\pi_{G}: S \rightarrow S / G$ and $\pi_{\operatorname{ker}\left(U_{i}\right)}: S \rightarrow S / \operatorname{ker}\left(U_{i}\right)$ coincide, and therefore the covering $\pi^{\operatorname{ker} U_{i}}: S / \operatorname{ker} U_{i} \rightarrow S / G$ is unramified. Computing Riemann-Hurwitz for this covering, we obtain that the genus of $S / \operatorname{ker}\left(U_{i}\right)$ is one.

In general, the Jacobian variety $J\left(S / \operatorname{ker} U_{i}\right)$ decomposes as the following product (see [3])

$$
J\left(S / \operatorname{ker} U_{i}\right) \sim \times_{j} B_{j}^{<\operatorname{Ind}_{\operatorname{ker} U_{i}}^{G} 1, U_{j}>} .
$$

In particular, $B_{i}$ appears in this decomposition with positive exponent. If the genus of $S / \operatorname{ker} U_{i}$ is 1 , all the subvarieties appearing on the decomposition of $J\left(S / \operatorname{ker} U_{i}\right)$ with positive exponent, except the one associated to the trivial representation, have dimension 0 (in particular $B_{i}$ ), completing the proof of the equivalences.

For the proof of the last statement, consider the natural epimorphism $\phi: G \rightarrow G / \operatorname{ker} U_{i}$. If $\left\{a, b, c_{1}, \ldots, c_{t}\right\}$ is a generating vector for $G$, then $\{\bar{a}:=\phi(a), \bar{b}:=\phi(b)\}$ is a set of generators for $G / \operatorname{ker} U_{i}$. Since $[\bar{a}, \bar{b}]=$ $\phi([a, b])=1, G / \operatorname{ker} U_{i}$ is abelian. Consider the representation $\overline{\theta_{i}}$ of $G / \operatorname{ker} U_{i}$ determined by $\theta_{i}$ (the representation afforded by $U_{i}$ ); i.e., for $\bar{k} \in G / \operatorname{ker} U_{i}$ define $\overline{\theta_{i}}(\bar{k})=\theta_{i}(k)$, where $k$ is a representative for $\bar{k}$. It is a well defined representation; moreover, it is irreducible if and only if $\theta_{i}$ is, and its degree is the same as the degree of $\theta_{i}$. As $G / \operatorname{ker} U_{i}$ is abelian, the degree of $\theta_{i}$ is 1 . 
Example 5.14. Consider the group $G=\mathbb{Z} / 4 \mathbb{Z}$, the cyclic group of order 4 , with generator $x$. $G$ has three rational irreducible representations: the trivial one $\theta_{0}$, another of degree one $\theta_{1}$, and $\theta_{2}$ of degree 2 .

It acts on a Riemann surface $S$ of genus 3 ([2, Table 5]), with signature $(1 ; 2,2)$. For this signature the only possibility for the stabilizer of points is $H=<x^{2}>$. Computing the intermediate covering for the subgroup $H$, we see that $S / H$ has genus one and $\pi^{H}: S / H \rightarrow S / G$ is a degree two unramified covering. Computing the dimension of the subvarieties on the isogeny decomposition of $J S$, we obtain that the dimension of the subvariety $B_{1}$ (corresponding to $\theta_{1}$ ) is 0 .

Theorem 5.10 states that the geometric signature for the action of a group $G$ on a Riemann surface $S$ determines the isotypical decomposition for the rational representation for the action of $G$ on the corresponding Jacobian variety. The converse is also true, as we show next.

Theorem 5.15. Let $S$ be a Riemann surface with $G$-action. Then the geometric signature of the action of $G$ uniquely determines the isotypical decomposition for the complexification of the rational representation for the action of $G$ on the corresponding Jacobian variety. Conversely, this decomposition uniquely determines the geometric signature for the action.

Proof. The forward implication is Theorem 5.10. Conversely, if we have two different geometric signatures, we know by Theorem 3.7 that the genera of the intermediate quotients by the cyclic subgroups of $G$ are different in at least one case. Considering (5.3), we have the same matrix $\Omega$ but different values of $g_{S / H_{j}}$. Thus, both solutions must be different.

Example 5.16. Continuing with Example 4.3, we want to show that the two different injections of $G$ into $\operatorname{Aut}(S)$ give two different decompositions for the rational representation associated to the action of $W C_{3}$ on the Jacobian variety corresponding to $S$. Therefore, there are two different ways of describing the subvarieties appearing in the isogeny decomposition of $J S$.

$W C_{3}$ has ten rational irreducible representations, all of them are absolutely irreducible. Computing the multiplicity of each one in the isotypical decomposition of $\rho_{\mathbb{Q}} \otimes \mathbb{C}$ by using Theorem 5.10, we find that in the first case just one representation, $\theta_{1}$, has multiplicity different from 0 in fact, the multiplicity is 2 . In the second case, again just one representation has multiplicity different from 0 (again, the multiplicity is 2), but it is $\theta_{2}$ in this case. The characters of these representations of $W C_{3}$ are as follows:

$$
\left[\begin{array}{ccccccccccc} 
& \mathrm{Id} & x y z & x y & x y z b & z y b & x & z y a b & x y z a^{2} & y z a^{2} & z a b \\
\theta_{1} & 3 & -3 & -1 & -1 & -1 & 1 & 1 & 0 & 0 & 1 \\
\theta_{2} & 3 & -3 & -1 & 1 & 1 & 1 & -1 & 0 & 0 & -1
\end{array}\right]
$$


If we compute the dimensions of the respective components of the isogeny decomposition of the corresponding Jacobian variety, we find that in both cases the corresponding $B_{j}$ (cf. Theorem 2.1) has complex dimension one. Therefore, in both situations $J S$ is isogenous to a product of three elliptic curves. In the first situation $G$ acts through the representation $\theta_{1}$, and in the other one through $\theta_{2}$.

\section{References}

[1] Breuer, T.: Characters and Automorphism Groups of Compact Riemann Surfaces. London Mathematical Society Lecture Note Series 280. Cambridge University Press, 2000.

[2] Broughton, S. A.: Classifying finite group actions on surfaces of low genus. J. Pure Appl. Algebra 69 (1991), no. 3, 233-270.

[3] Carocca, A. And Rodríguez, R.: Jacobians with group actions and rational idempotents. J. Algebra 306 (2006), no. 2, 322-343.

[4] Curtis, C. And Reiner, I.: Representation theory of finite groups and associative algebras. Pure and applied mathematics XI. Interscience Publishers, New-York-London, 1962.

[5] Farkas, H. And Kra, I.: Riemann Surfaces. Graduate Texts in Mathematics 71. Springer-Verlag, New York, 1991.

[6] G.A.P. Groups, Algorithm and Programming Computer Algebra System. http://www.gap-system.org/ gap

[7] Jones, G. A. And Singerman, D.: Complex functions: An algebraic and geometric viewpoint. Cambridge University Press, Cambridge, 1987.

[8] Ksir, A.: Dimensions of Prym Varieties. Int. J. Math. Math. Sci. 26 (2001), no. $2,107-116$.

[9] Lange, H. And Recillas, S.: Abelian Varieties with group action. J. Reine Angew. Math. 575 (2004), 135-155.

[10] Miranda, R.: Algebraic Curves and Riemann Surfaces. Graduate Studies in Mathematics 5. American Mathematical Society, Providence, 1995.

[11] Recillas, S. and Rodríguez, R.: Jacobians and Representations for $S_{3}$. In Workshop on Abelian Varieties and Theta Functions (Morelia, 1996), 117-140. Aportaciones Mat. Investig. 13. Soc. Mat. Mexicana, México, 1998.

[12] Rojas, A. M.: Group actions on Jacobian varieties. Ph.D. Thesis. Pontificia Universidad Católica de Chile, 2002.

[13] SÁnchez-Argáez, A.: Acciones de $A_{5}$ en Jacobianas de curvas. Aportaciones Mat., Comun. 25 (1999), 99-108.

[14] Serre, J. P.: Linear Representations of Finite Groups. Graduate Texts in Mathematics 42. Springer, 1996. 
[15] Singerman, D.: Subgroups of Fuchsian groups and finite permutation groups. Bull. London Math. Soc. 2 (1972), 319-323.

[16] Streit, M.: Period Matrices and Representation Theory. Abh. Math. Sem. Univ. Hamburg 71 (2001), 279-290.

[17] Suzuki, M.: Group Theory I. Grundlehren der Mathematischen Wissenschaften 247. Springer-Verlag, Berlin-New York, 1982.

[18] Völklein, H.: Groups as Galois groups. Cambridge studies in Advanced Mathematics 53. Cambridge University Press, 1996.

[19] Wolfart, J.: Regular dessins, endomorphisms of Jacobians, and transcendence. In A Panorama of Number Theory or The View from Baker's Garden (Zürich, 1999), 107-120. Cambridge University Press, Cambridge, 2002.

[20] Wolfart, J.: Triangle groups and Jacobians of CM type. Manuscript, Frankfurt a.M., 2000. http://www.math.uni-frankfurt.de/ wolfart.

Recibido: 13 de octubre de 2003

Revisado: 11 de octubre de 2005

Anita M. Rojas

Departamento de Matemáticas

Facultad de Ciencias

Universidad de Chile

Las Palmeras 3425, Ñuña

Santiago, Chile.

anirojas@uchile.cl

Finished with the partial support of FONDECYT No. 3040066. 